\title{
Co-evaluación de Competencias en el Proyecto Final de Carrera: Aplicación a la Titulación de Administración y Dirección de Empresas
}

\author{
Harold R. Torrez, Marta Tena y Lucinio González \\ Universitat Ramon Llull, Facultad de Economía IQS, Departamento de Gestión Empresarial, \\ Vía Augusta, 390, 08017 Barcelona, España (e-mail: haroldtorrezm@iqs.url.edu; \\ marta.tena@iqs.edu; lucinio.gonzalez@iqs.edu)
}

Recibido May. 17, 2011; Aceptado Jun. 13, 2011; Versión final recibida Jul. 03, 2011

\begin{abstract}
Resumen
El presente trabajo muestra una metodología basada en la recolección de información de estudiantes y tutores relacionados con las competencias que se desarrollan en el Proyecto Final de Carrera en la titulación de Administración y Dirección de Empresas de la Facultad de Economía IQS de la Universidad Ramon Llull, España. Se aplicó una encuesta para determinar el nivel de mejora en las competencias, mediante una auto-evaluación por parte de los estudiantes y una evaluación por parte de los tutores. Los resultados permiten afirmar que ambas evaluaciones tienen un componente de similitud. Las futuras líneas de investigación que abren este trabajo se dirigen en la comparación de los resultados de un año al otro para conocer las variaciones existentes e introducir los cambios que sean necesarios para mejorar las competencias de los estudiantes.
\end{abstract}

Palabras clave: evaluación, auto-evaluación, competencias, proyecto final de carrera

\section{Co-evaluation of skills in the Final Project of Undergraduate Studies: The Case of the Graduation in Business Administration}

This paper shows a methodology based on gathering information from students and tutors related with the development of skills in the Final Project of the career Business Administration at the IQS School of Management, Ramon Llull University, Spain. A survey was applied to determine the level of skill improvement through self-assessment tests by students and evaluation by the advisors. The results confirm that both evaluations (students and advisors) are similar. Future research in this area should be aimed to comparing the results from one year to another to become familiar with variations in time, and to introduce the changes that are necessary to improve skills in students.

Keywords: assessment, self-assessment, skills, final project 


\section{INTRODUCCIÓN}

El marco educativo universitario en la actualidad se enfrenta a cambios profundos en sus bases fundamentales, ya que el proceso de integración en el Espacio Europeo de Educación Superior (EEES), ha generado rápidamente transformaciones importantes en el proceso de aprendizaje, ya que se busca sintonizar y alcanzar puntos de referencia a nivel europeo en la educación; (TunningEducationalStructures in Europe, 2003). Esto quiere decir que, tanto docentes como estudiantes y otros agentes que facilitan el aprendizaje deben reestructurar su manera de percibir la enseñanza. Una de las novedades que ha supuesto el desarrollo del EEES es la necesidad de que el estudiante desarrolle competencias que faciliten su empleabilidad una vez acabados los estudios; por lo tanto se añade a los objetivos docentes tradicionales, el desarrollo de competencias por parte de los estudiantes y su evaluación.

Por otra parte, se ha hecho obligatoria la realización de un Proyecto Final de Carrera (PFC) como elemento final de síntesis de todo lo estudiado durante el periodo de formación universitaria de Grado, la realización del PFC no sigue la estructura clásica de las demás asignaturas, a diferencia, es un trabajo a realizar, en pareja o grupo de tres, durante el último año, bajo la dirección de un tutor, sin horario académico estricto, pero con plazos de entrega establecidos. En el caso del Grado en Administración y Dirección de Empresas al que se hace referencia en este trabajo, el proyecto consiste en desarrollar un plan de empresa viable y novedosa en alguna medida. Por lo tanto, es una buena oportunidad para aplicar los conocimientos adquiridos y las competencias desarrolladas durante los estudios; y en consecuencia es necesario evaluar ambos aspectos. Tradicionalmente, el proyecto se evaluaba mediante su defensa ante un tribunal, desde la implantación de las nuevas titulaciones de Grado en el marco del EEES, es necesario evaluar también el nivel de competencias adquirido.

Antes de tratar la evaluación de las competencias, consideramos interesante recoger el concepto de competencia de las numerosas aportaciones sobre dicho concepto, para el marco del presente trabajo se tomará en cuenta las definiciones de Royo y del Cerro (2005), y Goñi (2005), los cuales indican que las competencias son saberes, rasgos, aptitudes, actitudes, valores, conocimientos, habilidades y capacidades, que permiten desarrollar un adecuado rendimiento en un puesto laboral. Además éstas características de las personas se ponen en evidencia cuando se lleva a cabo un trabajo, pero que además se relacionan con la ejecución exitosa de la actividad. Por otra parte es importante definir el proceso de evaluación; Eklöf (2010) y Tanggaard, Elmholdt, y Claus (2008)indicanque un proceso de evaluación simplificado y directo, consta de cuatro fases; en primer lugar está el propósito de la evaluación, en segundo lugar se encuentra la calidad del instrumento y el procedimiento de evaluación, como tercer elemento se interpreta los resultados y por último se discuten los datos obtenidos para ver si son válidos y generan información pertinente.

Un dispositivo de evaluación fiable debe ir acompañado de un cambio en el comportamiento docente, en los planes de estudio y en la didáctica (Griffin et al. 2010). Cada profesor deberá ser consciente del aprendizaje de cada estudiante, por lo tanto, la teoría y práctica en una asignatura debe responder a una estrategia de aprendizaje global que mediante el uso de ciertos recursos sirven como elementos de motivación. KatrienStruyven, FilipDochy y Steven Janssens (2005)interpretan la evaluación como un proceso que sirve de apoyo en la toma de decisiones racionales, por lo tanto la evaluación debe ser útil y proponer informes periódicos que faciliten procedimientos fiables y transparentes, respondiendo a un componente ético que asegure la honestidad de la información y evite subjetividades al presentarla.

Cuando nos referimos a la autoevaluación, es preciso definirla adecuadamente; Ducasse (2004) relaciona a la autoevaluación como un componente en el aprendizaje, que hace resaltar las metas personales del estudiante y permite identificar el progreso que ha obtenido a lo largo de un tiempo determinado. McDonald y Boud (2003), y Ross (2006), dan un paso más e indican que la autoevaluación es la estrategia por excelencia para educar en la responsabilidad, sobre todo aprendiendo a valorar, criticar y reflexionar sobre el proceso de aprendizaje individual realizado por el estudiante, Cassidy (2007), indica que es importante llevarla a cabo con frecuencia, ya que 
de esta manera se rompe con las estructuras clásicas de educación, Hosal-Akman y SimgaMugan (2010) y Hyvärinen et al. (2011), complementan haciendo referencia a la importancia que tiene la capacitación de los evaluadores, ya que mediante dicha capacitación se intentará asegurar un acuerdo sobre la escala de valoración para que exista coherencia y similitud entre las evaluaciones de los tutores y de los estudiantes.

McDonald (2009) aporta los resultados que ha obtenido en una investigación, donde llega a la conclusión de que la autoevaluación parte de un entrenamiento, y hace que las personas que lo aplican desarrollen capacidades reflexivas, introspectivas, analíticas, críticas y autónomas. En su estudio demuestra que los estudiantes que se formaron mediante la autoevaluación, se sienten más preparados para tomar decisiones y encontrar soluciones a problemas cotidianos y profesionales. Este resultado está en línea con el publicado por Leach (2010) que concluye en su estudio que las evaluaciones entre estudiantes y profesores son similares, con una correlación estadísticamente muy significativa, pero además destaca que los estudiantes de mayor rendimiento tienden a calificarse de manera más baja que los estudiantes normales quienes sobreestiman su desempeño.

Aunque la evaluación de las competencias dentro el Proyecto Final de Carrera es un tema de carácter novedoso y desafiante,Savin-Baden y Howell (2004), indican que los trabajos enfocados en un aprendizaje basado en proyectos, y que además sea cooperativo, aporta ciertos elementos que hace que los estudiantes desarrollen capacidades para afrontar momentos de incertidumbre, planificar sus actividades y por último tomen decisiones basadas en un proceso de razonamiento lógico. Aragonés y Luján (2005), López et al. (2007), muestran su apertura a la implementación de proyectos en la universidad, ya que según ellos sirve de ayuda a la hora de generar una evaluación continua, ya sea mediante entregas parciales u otro método de evaluación, además Costley (2007), indica que estas teorías de aprendizaje permiten a los estudiantes basados en proyectos reflexionar críticamente sobre su aprendizaje en el trabajo, lo cual les permite justificar las pretensiones de su aprendizaje y aportar pruebas.

En el estudio realizado por Velasco y Villavieja (2009), se muestra un problema en la evaluación tradicional de los proyectos, que se refiere a la evaluación única por parte del profesor, ellos indican que no es suficiente conocer la evaluación de un profesor; los estudiantes tienen tutores u otros agentes que también pueden aportar información para generar una evaluación más certera que agrupe los diferentes criterios que tienen las personas responsables de la formación de los estudiantes. Cabe destacar que las aportaciones que existen en la evaluación de competencias en el Proyecto Final de Carrera, básicamente se enfocan en el ámbito de las ciencias exactas o ingenierías; en este contexto, Jiménez et al. (2009), muestran estudios que evalúan las competencias mediante algoritmos que miden los logros que han tenido los estudiantes. El objetivo del presente trabajo ha sido la evaluación de competencias adquiridas a través del Proyecto Final de Carrera de una titulación universitaria. Han participado los propios estudiantes (autoevaluación) cuya opinión se ha comparado con la de sus tutores; ambos valoraron el nivel adquirido de cada competencia por los estudiantes a lo largo del proceso de elaboración del PFC. La opinión de los tutores ayuda a validar la percepción manifestada por los estudiantes acerca del nivel de competencias que han podido adquirir durante el periodo dedicado al PFC y como consecuencia de su realización.

\section{METODOLOGÍA}

EI PFC se realiza en el último curso de la titulación, en este caso, Administración y Dirección de Empresas. El proyecto consiste en la presentación de un plan de empresa, donde se muestra la viabilidad de la misma. Con el proyecto se puede evaluar la capacidad de llevar a la práctica la mayoría los conocimientos que el estudiante debería haber adquirido a lo largo de su proceso formativo y ofrece una visión del logro de las competencias desarrolladas al final del programa. La organización del PFC, se inicia con la formación de parejas o grupos de tres estudiantes, a continuación se selecciona la idea de negocio que se pretende realizar, esto en coordinación con los profesores de la asignatura, posteriormente se redacta un Briefing de 3 a 5 páginas donde se explica la idea de negocio, el producto o servicio que se pretende llevar a cabo y las motivaciones 
y posibles limitaciones que pueden tener. Teniendo el Briefing de los estudiantes, los profesores de la asignatura se reúnen con los tutores externos quienes son personas que provienen del ámbito empresarial, y que sólo dedican las horas de tiempo para tutorizar a los estudiantes; (no ejercen como docentes), por lo tanto y de cara a la formación de los estudiantes, el contacto directo con gente de empresa hace que se complemente el conocimiento que los estudiantes tenían al iniciar sus estudios universitarios. Cabe destacar que dentro del equipo de tutores (14 tutores) se encuentran una mezcla de conocimientos en diferentes sectores empresariales, por lo tanto la asignación de una idea de negocio se hace mediante la familiaridad que puede tener un tutor respecto a un tema, de esta manera se garantiza que los trabajos puedan contar con mayor calidad. El profesor de la asignatura fija plazos de entrega periódicos, con la supervisión de los tutores, quienes son los directos responsables del avance de los proyectos hasta la presentación definitiva de la memoria. El proyecto se defiende ante un tribunal examinador conformado por el Director o Decano, un profesor de la universidad y un invitado experto del entorno empresarial.

Para fines investigativos, se tomó la decisión de no utilizar un grupo de control, ya que la estructura física y académica que tiene la universidades bastante familiar, por lo tanto lo que busca el trabajo es conocer las competencias que todos los estudiantes han adquirido durante la realización del PFC,lo cual imposibilita la opción de separarlos por grupos de estudio, en este sentido la estrategia que se aplica es valorarlos en igualdad de condiciones que sus demás compañeros. Desde la perspectiva ética, no sería correcto tener distinciones en la calidad de la enseñanza, por tal razón los tutores ingresan al terreno de juego, ya que ellos serán pieza fundamental para evitar subjetividades en las autoevaluaciones.

El cuestionario de evaluación se estructuró mediante 17 preguntas que hacen referencia directa a las competencias que los estudiantes han podido desarrollar, cabe destacar que el método de administración de la encuesta fue personal y libre, los estudiantes debían poner su nombre y autoevaluarse en cada una de las preguntas, la muestra que existía en ese momento fue de 100 personas matriculadas en el PFC, pero al ser libre algunas de ellas prefirieron no responder al cuestionario.

Para estimar el nivel de competencias adquirido al final de los estudios, se analizaron las opiniones que los estudiantes manifestaron respecto a su percepción del nivel adquirido para cada una de las 17 competencias recogidas en la tabla 1. Todas las preguntas tenían la misma escala ordinal de 6 niveles: 1: nada; 2: poco; 3: algo; 4: bastante; 5: mucho; y 6: muchísimo.

Cabe destacar que la evaluación de 17 competencias responde a una estrategia de control, inicialmente se encuentran divididas en transversales que son obligatorias para la asignatura (trabajo en equipo, análisis, síntesis, TICs, creatividad, innovación y comunicación oral), pero también se agregaron otras que son específicas y sirven como preguntas de control y de comparación, ya que permiten saber si las respuestas que se obtienen son consistentes con las competencias que los profesores y tutores motivan a los estudiantes durante todo el desarrollo del PFC. Por una parte, se invitó a los estudiantes antes de presentar su PFC, a responder al cuestionario donde señalaron el nivel adquirido en cada una de las competencias como resultado de realizar el proyecto. De esa manera realizaron su propia autoevaluación. Por otra parte, se pidió a sus tutores que respondieran al mismo cuestionario, con las preguntas adecuadamente redactadas, para indicar su opinión sobre el nivel adquirido por sus tutorizados al llegar al final del proyecto y de esta forma validar las respuestas de los estudiantes.

Se procesaron las encuestas de todos los estudiantes y se obtuvo la valoración media de cada competencia. Se estandarizaron las medias linealmente en una escala de 0 a 100, tomando como origen la media de la respuesta a la pregunta 8 (nivel de inglés) puesto que no se había exigido ni promocionado ninguna actividad en este idioma, y como máximo de la escala la media de la respuesta a la pregunta 15 (desarrollar un Plan de Empresa) ya que este era el objetivo principal de la asignatura de PFC y se consideró que todas las demás competencias podrían mejorar tanto o más que la 8 y como mucho, tanto como la 15. De esta forma quedó construida una escala interna para la ordenación del nivel de mejora de cada competencia. El mismo proceso se aplicó todas las encuestas contestadas por los tutores. 
Tabla 1: Cuestionario sobre competencias

\begin{tabular}{|c|l|}
\hline 1 & ¿Ha mejorado tu capacidad para trabajar en equipo? \\
\hline 2 & ¿Ha mejorado tu capacidad de análisis? \\
\hline 3 & ¿Ha mejorado tu capacidad para trabajar en el entorno financiero? \\
\hline 4 & ¿Ha mejorado tu capacidad para trabajar en un entorno de marketing y ventas? \\
\hline 5 & $\begin{array}{l}\text { ¿Has perfeccionado tu habilidad en el uso de las tecnologías informáticas para gestionar } \\
\text { información? }\end{array}$ \\
\hline 6 & ¿Has perfeccionado tu habilidad para comunicar resultados por escrito? \\
\hline 7 & ¿Ha mejorado tu habilidad para desarrollar ideas innovadoras? \\
\hline 8 & ¿Ha mejorado tu nivel de inglés? \\
\hline 9 & ¿Ha mejorado tu capacidad para realizar estudios de mercado? \\
\hline 10 & $\begin{array}{l}\text { ¿Ha mejorado tu capacidad para trabajar de acuerdo a un calendario de fechas de } \\
\text { presentación? }\end{array}$ \\
\hline 11 & ¿Ha mejorado tu capacidad de síntesis? \\
\hline 12 & ¿Ha mejorado tu habilidad de liderazgo? \\
\hline 13 & $\begin{array}{l}\text { ¿Ha mejorado tu habilidad en el uso de las tecnologías informáticas para buscar } \\
\text { información? }\end{array}$ \\
\hline 14 & ¿Ha mejorado tu capacidad para percibir los cambios que se producen en el entorno? \\
\hline 15 & ¿Ha mejorado tu capacidad para desarrollar un Plan de Empresa? \\
\hline 16 & ¿Ha mejorado tu capacidad creativa? \\
\hline 17 & $\begin{array}{l}\text { ¿Ha mejorado tu capacidad para realizar elecciones éticamente fundamentadas en el } \\
\text { ámpresarial? }\end{array}$ \\
\hline
\end{tabular}

\section{RESULTADOS}

Tras una prueba piloto inicial,la cual se aplicó de forma no anónima a 10 estudiantes, con la finalidad de conocer si la manera de redacción era adecuada para hacer este tipo de preguntas relacionadas a las competencias, se determinó que los estudiantes participantes de la prueba piloto comprendieron claramente el fin de la investigación, ya que al terminar de responder el cuestionario se les hizo una rápida entrevista sobre las dificultades o malentendidos que pudieron tener al responder las preguntas, al no existir ninguna dificultad, se decidió no realizar cambio alguno para la prueba definitiva. La encuestadefinitiva se llevó a cabo a 68 estudiantes de Administración y Dirección de Empresas que presentaron su PFC durante Junio de 2010 en la Facultad de Economía IQS de la UniversitatRamonLlull (Barcelona, España). Los estudiantes fueron evaluados por sus tutores, participando como tales 14 profesores diferentes. En total, 30 proyectos presentados realizados en grupos de 2 ó 3 estudiantes. La figura 1 muestra los resultados obtenidos en la evaluación realizada por los tutores y la autoevaluación de los estudiantes. Se ha utilizado la media de las respuestas en una escala de 1 a 6 y cabe destacar que no se aprecian grandes diferencias en la evaluación de las diferentes competencias en función del autor de la evaluación, por lo que la percepción que tienen los estudiantes sobre el logro de cada una de las competencias es coherente con la evaluación realizada por los tutores. Este resultado está en línea con el publicado por Leach (2010) ya citado. En la figura 1 se puede apreciar que la valoración de los tutores es más alta que la de los estudiantes, esto puede deberse a que los estudiantes al realizar una autoevaluación tienden a evaluarse a la baja, mientras que los tutores reflejan de manera objetiva su valoración ya que ellos son parte del proceso de confección del proyecto de empresa y son los directos responsables de una adecuada formación y presentación de un trabajo. 


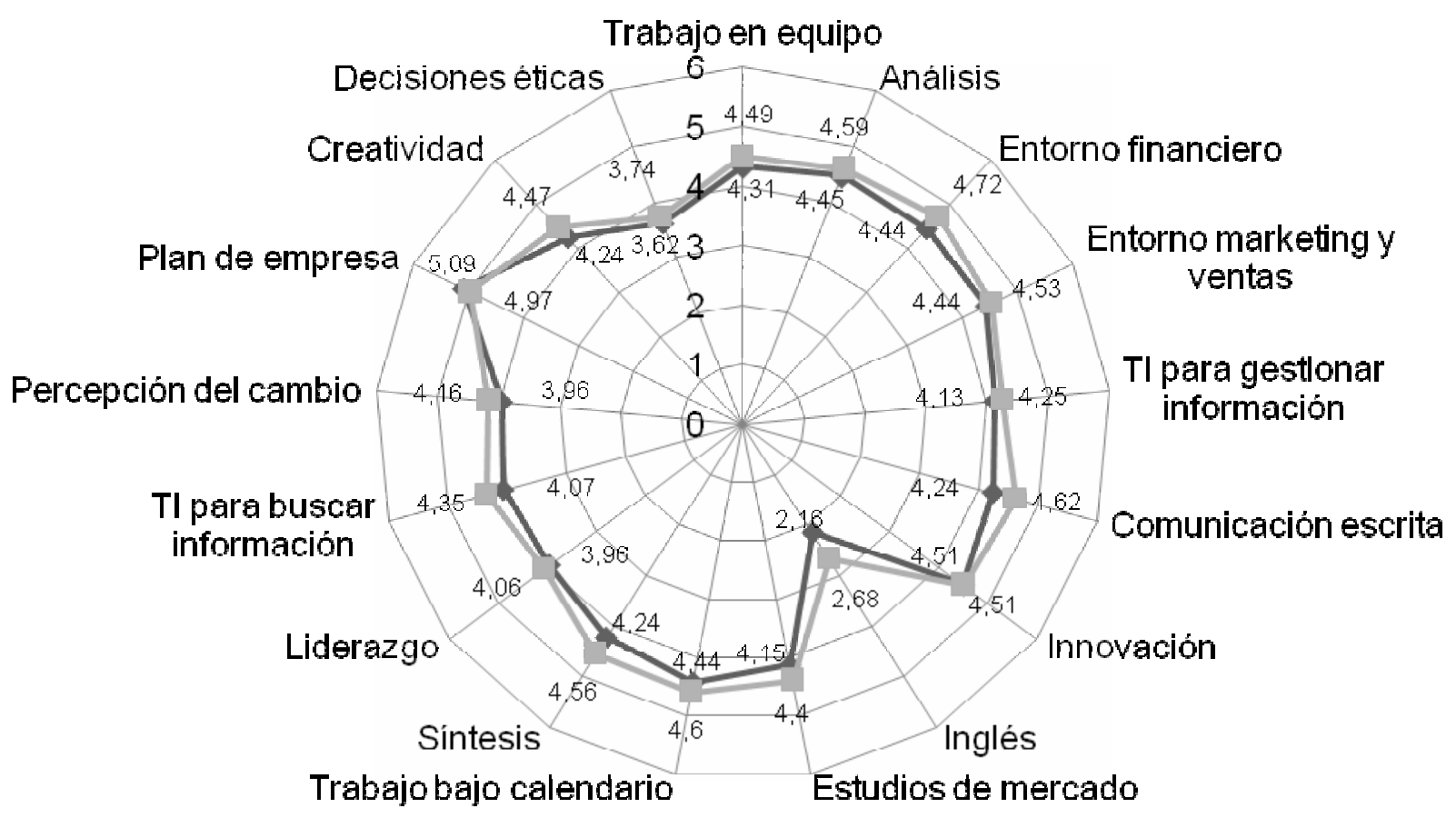

Fig. 1: Medias de estudiantes y tutores

Una vez normalizadas las medias tal como se ha descrito más arriba, se obtiene la figura 2. Se observa que todas las competencias tienen una media dentro de la escala. Visto desde la perspectiva más positiva, la habilidad de realizar el Plan de Empresa es la competencia con un nivel de mejora más alto mientras que la mejora del nivel de inglés representa el mínimo. Esta estandarización permitirá disponer de criterios de comparación para promociones futuras dentro de un proceso de mejora continua ya que serán evaluados con ésta misma herramienta y metodología, de manera que cuando se incida sobre la mejora de alguna competencia, esta mejora se deberá ver reflejada en un incremento porcentual de un año al otro.

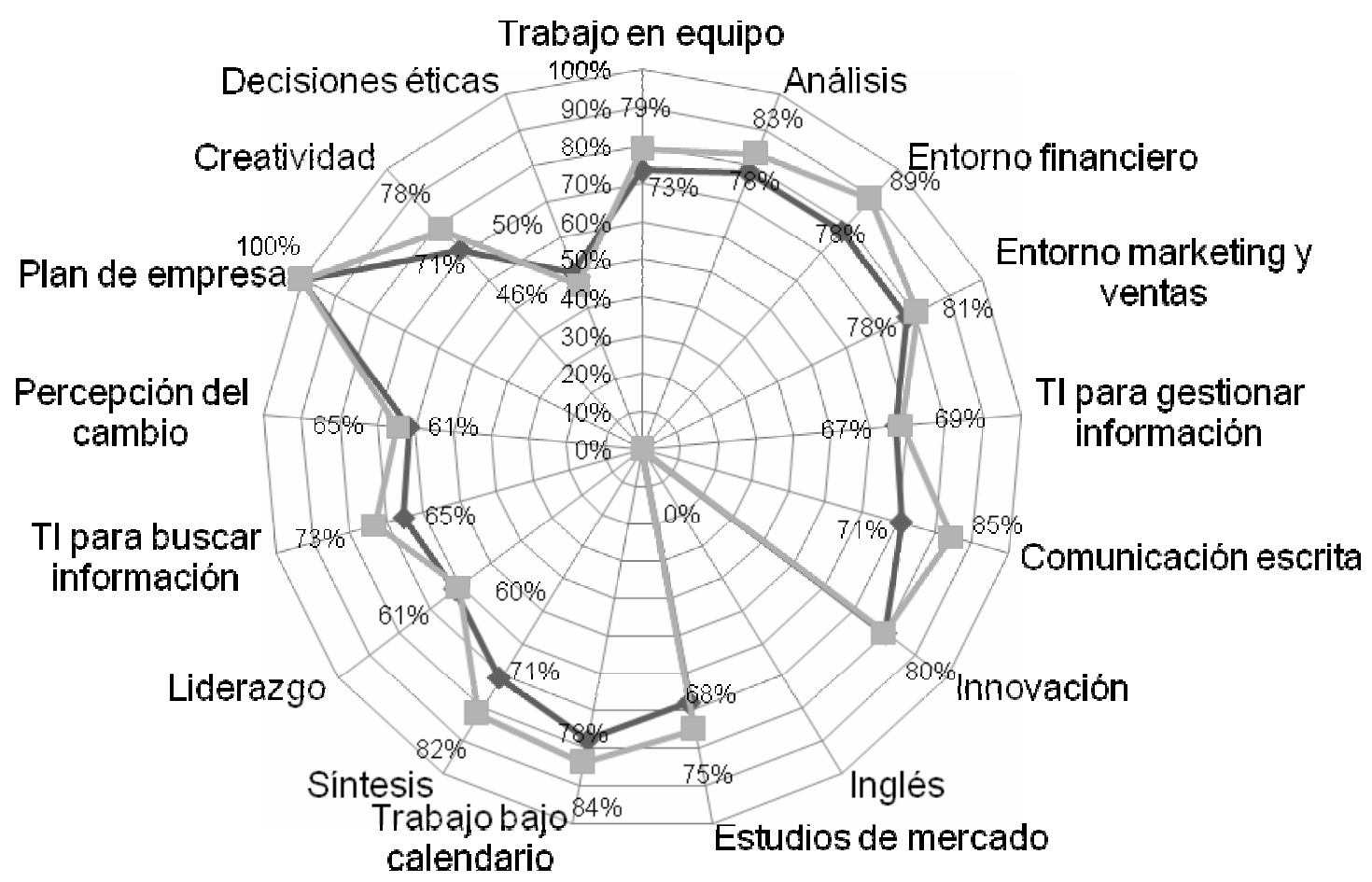

Fig. 2: Medias normalizadas de tutores y estudiantes 


\section{CONCLUSIONES}

Las competencias desarrolladas en el Proyecto Final de Carrera son evaluadas por los estudiantes positivamente ya que éstos son plenamente conscientes de haber desarrollado habilidades y del progreso de las mismas. En consecuencia, la puntuación obtenida es satisfactoria. Además, la evaluación realizada por los tutores respecto al logro de las competencias de los estudiantes es coherente con la realizada por los estudiantes por lo que se observa congruencia en los resultados obtenidos.

La aplicación de la metodología de evaluación de competencias descrita en los Proyectos Finales de Carrera de la titulación es innovadora en el ámbito de las ciencias sociales. Dicha metodología representa un paso inicial de generación de cambio en el comportamiento de los agentes que intervienen en el proceso de evaluación, promueve un proceso de aprendizaje en la misma realización de la evaluación y sirve de soporte para implementar en el proceso evaluativo la coevaluación.

El presente trabajo abre nuevas líneas de investigación. Entre las mismas, destacamos el estudio comparativo de los resultados obtenidos a lo largo de los años con el objetivo de analizar el progreso de los estudiantes en el logro de las competencias. Por otra parte, permite detectar áreas de mejora en determinadas competencias posibilitando que el profesorado realice el refuerzo oportuno en las mismas.

\section{REFERENCIAS}

Aragonés F. J. y Luján-Mora, S., Los créditos ECTS en la enseñanza de la asignatura Programación en Internet, Investigar en diseño curricular: Redes de docencia en elespacio europeo de educación superior, (1), 325-350 (2005).

Calatayud, A., La cultura autoevaluativa, piedra filosofal de la calidad en educación, Educadores: Revista de la Federación Española de Religiosos de Enseñanza(204), 357-375 (2002).

Cassidy, S., Assessing 'inexperienced' students' ability to self-assess: exploring links with learning style and academic personal control, Assessment \& Evaluation in Higher Education, 32(3), 313330 (2007).

Costley, C., Work-based learning: assessment and evaluation in higher education, Assessment \& Evaluation in Higher Education, 32(1), 1-9 (2007).

Ducasse, A., La autoevaluación como parte de la nota semestral. Revista redELE. ISSN: 15714667 (en línea), 2004. http://www.educacion.es/redele/revista/ducasse.shtml. Acceso: 31 de Marzo (2011).

Eklöf, H., Skill and will: test-taking motivation and assessment quality, Assessment in Education: Principles, Policy \& Practice, 17(4), 345-356 (2010).

Fuentes, M. y J. Herrero, Evaluación Docente: hacia una fundamentación de la autoevaluación. Revista Electrónica Interuniversitaria de Formación del Profesorado, ISSN 1575-0965, (en línea) 2(1), 353-368, (1999). http://www3.uva.es/aufop/publica/actas/ix/32-fuentes.pdf. Acceso 31 de Marzo (2010).

Goñi, J.M., El espacio europeo de educación superior, un reto para la universidad. Competencias, tareas y evaluación, los ejes del currículum universitario. Octaedro/ICE-UB. Barcelona, España (2005).

Griffin, P., Murray, L., Care, E., Thomas, A. y Perri, P., Developmental assessment: lifting literacy through professional learning teams, Assessment in Education: Principles, Policy \& Practice, 17(4), 383-397 (2010). 
Hosal-Akman, N. y Simga-Mugan., Can an assessment of the effects of teaching methods on academic performance of students in accounting courses, Innovations in Education and Teaching International, 47(3), 251-260 (2010).

Hyvärinen, M., Tanskanen, P., Katajavuori, N. y Isotalus, P. Evaluating the use of criteria for assessing profession-specific communication skills in pharmacy, Studies in Higher Education, First published on: 26 April 2011 (iFirst) (2011).

Jiménez, F., G. Martínez, G. Sánchez, J. M. Juárez, y S. Paredes, Un sistema de evaluación basado en competencias: Ejemplo para la asignatura Tecnología de la Programación del título de grado en Ingeniera Informática por la Universidad de Murcia. Actas de las XV Jornadas de Enseñanza Universitaria de Informática, JENUI 2009, 193-200, Barcelona, España, 8 al 10 de Julio (2009).

Katrien, S., Filip, D. y Steven, J. Students' perceptions about evaluation and assessment in higher education:a review, Assessment \& Evaluation in Higher Education, 30(4), 331 - 347 (2005).

Leach, L., Optional self-assessment: some tensions and dilemmas, Assessment \& Evaluation in Higher Education, First published on: 18 November 2010 (iFirst) (2010).

López, D., A. Pajuelo, J.R. Herrero, y A. Duran, Evaluación continuada sin morir en el intento, JENUI 07, 171-178, Teruel, España, 16 al 18 de Julio (2007).

McDonald, B. y Boud, D., The Impact of Self-assessment on Achievement: The effects of selfassessment training on performance in external examinations, Assessment in Education: Principles, Policy \& Practice, 10(2), 209-220 (2003).

McDonald, B., Exploring academic achievement in males trained in self-assessment skills, Education 3-13: International Journal of Primary, Elementary and Early Years Education, 37(2), 145 - 157 (2009).

Ovejero, A., El aprendizaje cooperativo: una alternativa eficaz a la enseñanza tradicional, Promociones y Publicaciones Universitarias, Barcelona, España (1990).

Ross, A.The Reliability, Validity, and Utility of Self-Assessment, Practical Assessment Research \&Evaluation, 11(10) (2006).

Royo, C. y A. del Cerro, Revisión teórica de las competencias: su origen, concepto y escuelas más importantes. ¿Hacia dónde se dirigen?, Psicología social y problemas sociales,1(4), 453-460 (2005).

Savin-Baden, M. y Howell, M., Foundations of problem-based learning. The society for research into higher education, McGraw-Hill Education, Maidenhead: Open University Press, ReinoUnido (2004).

Tanggaard, L.y Elmholdt, C. Assessment In Practice: An inspiration from apprenticeship, Scandinavian Journal of Educational Research, 52(1), 97 - 116 (2008).

Tunning Educational Structures in Europe (2003).Educación y Cultura; Socrates.

Velasco, L. y C. Villavieja, Cómo evaluar continua e individualmente en asignaturas basadas en proyectos, Actas de las XV Jornadas de Enseñanza Universitaria de Informática, JENUI 2009, 347-354, Barcelona, España, 8 al 10 de Julio (2009). 